\title{
HIPERTEXTOS Y MAPAS CONCEPTUALES \\ EN AMBIENTES DE APRENDIZAJE \\ COLABORATIVO
}

\section{Oscar Hernán Fonseca Ramírez \\ Magíster en Tecnologías de la Información Aplicadas a la Educación.}

\begin{abstract}
Report of investigation for determine the incidence in the retention of concepts of two experimental conditions: first between the students who construct conceptual maps against which they observe the simulation of the map constructed by an expert and the second social condition of work: colaborativo against individual, of this combination four experimental groups determined themselves. The students made the study of the concept "Model of the Atom" in a hypertextual software.
\end{abstract}

\section{RESUMEN}

Informe de investigación que buscó determinar la incidencia en la retención de conceptos de dos condiciones experimentales: la primera entre los estudiantes que construyen mapas conceptuales contra los que observan la simulación del mapa construido por un experto y la segunda la condición social de trabajo: colaborativo contra individual, de esta combinación se determinaron cuatro grupos experimentales, los cuales realizaron el estudio del concepto Modelo del Átomo" en un ambiente hipertextual.

\section{INTRODUCCIÓN}

El presente trabajo es el producto de la investigación realizada para determinar la incidencia en la retención de conceptos bajo dos condiciones experimentales: la primera entre los estudiantes que construyen mapas conceptuales contra los que observan la simulación del mapa construido por un experto y la segunda la condición social de trabajo: colaborativo contra individual, de esta combinación se determinaron cuatro grupos experimentales, los cuales realizaron el estudio del concepto "Modelo del Átomo" en un ambiente hipertextual.

Con el desarrollo de la investigación se buscaba refinar una estrategia de diseño de materiales para la educación que sirva de soporte a maestros y estudiantes para el análisis y optimización de procesos de aprendizaje que puedan contribuir significativamente al mejoramiento de la calidad de la educación en todos los niveles.

Relacionando los planteamientos de la metacognición con el desarrollo de la investigación se puede decir que: 
"Una ventaja al utilizar mapas conceptuales es que se hace uso del metaconocimiento, porque los mapas conceptuales son instrumentos útiles para ayudar a los estudiantes a reflexionar sobre la estructura y el proceso de producción de su propio conocimiento" (Novak y Gowin 1984). Al crear la certeza de lo que el alumno sabe, él estará en la capacidad de transformar más fácilmente su vida escolar.

La construcción de mapas conceptuales como medio de aprendizaje frente a los procesos de enseñanza aprendizaje cobra un papel importante porque se fomenta el autoaprendizaje. Es decir que, se cimenta la certeza sobre el conocimiento. Una necesidad imperante del sistema educativo es generar estrategias en las que se fomente no solamente la adquisición de conocimientos por parte de los estudiantes, sino, que además permitan desarrollar estrategias metacognitivas que ayuden en la autorregulación de los procesos de adquisición del conocimiento, tarea que puede llevarse a cabo con el uso de los mapas conceptuales.

\section{PROBLEMA}

\subsection{Planteamiento del problema}

El problema de investigación se plantea bajo dos condiciones de trabajo tendientes a favorecer la retención de conceptos en el aprendizaje autónomo: La construcción de mapas conceptuales y los ambientes de aprendizaje colaborativo

Bajo las dos premisas planteadas surgen dos preguntas desde las que se desarrollan los problemas de la investigación:

¿La retención de conceptos será superior para los estudiantes que construyen mapas conceptuales frente a los estudiantes que observan la simulación de la construcción de los mapas conceptuales realizados por un experto en el estudio de un hipermedio sobre el tema "Modelo del Átomo"?

¿La retención de conceptos será superior para los estudiantes que trabajan colaborativamente frente a los que lo hacen individualmente en el estudio de un hipermedio sobre el tema "Modelo del Átomo"?

\subsection{Justificación del problema}

Se ha llegado a hallazgos importantes, es importante la implementación de estos en las aulas por las siguientes razones:

El aprendizaje autónomo es una condición esencial para que los individuos mantengan su capacidad productiva en a Sociedad del Conocimiento, donde el saber tiene niveles de obsolencia muy altos. En consecuencia, la escuela debe propiciar, no sólo el conocimiento objeto, sino también la metacognición que transfiere control sobre los procesos de aprendizaje al propio estudiante.

El aprendizaje colaborativo toma vigencia en una sociedad conectada por redes telemáticas que abren la posibilidad de experiencias enriquecedoras que involucran a varios individuos que se influyen mutuamente para incrementar su formación y saber. Esta es una dimensión e neurálgica del cambio educativo en el futuro próximo. Vale anotar a este respecto el desarrollo de la red de Participación Educativa que realiza la Secretaría 
Distrital de Educación de Bogotá, el programa conexiones de Antioquia y, en general los llamados Sistemas Nerviosos Digitales para La Educación.

\section{HIPÓTESIS}

\subsection{Primera Hipótesis}

Los estudiantes que trabajan colaborativamente obtendrán un puntaje superior en una prueba de retención de conceptos frente a los estudiantes que trabajan individualmente la temática expuesta en un hipermedio sobre el "Modelo del Átomo".

\subsection{Segunda Hipótesis}

Los estudiantes que construyen mapas conceptuales obtendrán mejores resultados en una prueba final de retención de conceptos frente a aquellos que observan la simulación de la construcción de los mapas conceptuales realizadas por un experto.

\section{MARCO TEÓRICO}

La investigación se desarrolla bajo dos condiciones de trabajo que tienen como finalidad favorecer el aprendizaje autónomo: la construcción de mapas conceptuales y el ambiente social de aprendizaje colaborativo, que se utilizarán en el estudio de un hipermedio sobre "Modelo del átomo" (dominio de conocimiento en el área de química), sobre los que se evaluará estas condiciones en la retención de conceptos y que se encadenan en el gráfico 1.

\section{APRENDIZAJE AUTÓNOMO}

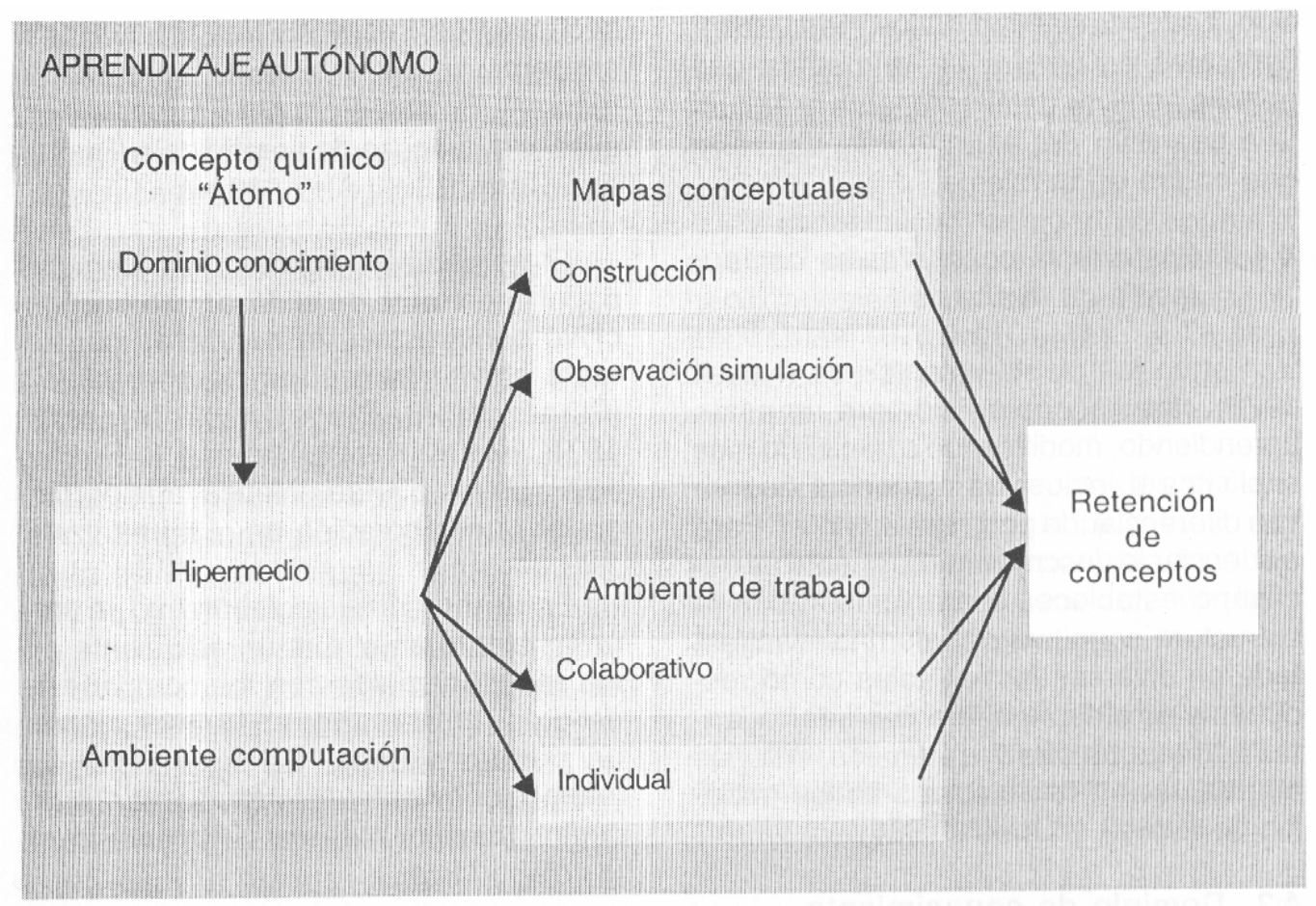

Gráfica 1. Modelo teórico de la investigación. 


\subsection{Mapas conceptuales}

Los mapas conceptuales son representaciones de conocimiento declarativo que tienen por objeto presentar las relaciones significativas entre conceptos en forma de proposiciones. Una proposición consta de dos o más términos conceptuales unidos por palabras para formar una unidad semántica (Novak 1988).

La mayor parte de los significados conceptuales se aprende mediante la composición de proposiciones en las que se incluye el concepto que se va a adquirir. Un mapa conceptual es un recurso esquemático para representar un conjunto de significados conceptuales incluidos en una estructura de proposiciones.

Los mapas conceptuales son una guía que sirve a los estudiantes para crear el camino más coherente en la construcción de una proposición nueva producto del enlace de conceptos. Los mapas conceptuales deben ser jerárquicos, y esta jerarquía va desde los elementos más generales e inclusivos hacia los elementos más particulares y menos inclusivos.

\subsubsection{Origen de los mapas conceptuales}

Tolman en 1932 publicó su libro "purposive behavior in animals and men" del cual se destaca que: "no se aprenden respuestas sino representaciones 'mapas cognitivos' acerca del ambiente". La noción de mapa cognoscitivo se retomó anos después cuando Linch (Linch 1960) infiere sus características en estudios acerca de la manera como las personas se representaban su ciudad. Otros autores le atribuyen funciones de orientación espacial que influyen en el razonamiento y la solución de problemas (Down y Stea 1977; Down 1981, en de Vega, 1984)

\subsubsection{Funcionalidad de los mapas conceptuales}

El concepto principal de la teoría constructivista es el aprendizaje significativo propuesto por Ausubel y el cual se puede corroborar a partir de la siguiente expresión: "Para aprender significativamente, el individuo debe relacionar los nuevos conocimientos con los conceptos y las proposiciones relevantes que ya se conocen" (Ausubel 1978). Según lo anterior, se requieren de instrumentos que pongan de manifiesto las relaciones significativas entre conceptos en forma de proposiciones.

A través de los mapas conceptuales se puede determinar el cambio conceptual dado mediante un aprendizaje significativo de conceptos por parte del alumno. Lo anterior se fundamenta en los planteamientos y resultado de las investigaciones de (Novak y Gowin, 1984) quienes encontraron que una vez completada una tarea de aprendizaje, los mapas conceptuales proporcionan un resumen esquemático de todo lo que se ha aprendido. La funcionalidad de los mapas conceptuales es amplia en la medida que permite analizar detalladamente la estructura cognitiva del alumno y la dinámica que ocurre en la misma.

\section{1.3 Los mapas conceptuales como medio de evaluación}

Según los planteamientos de (Novak 1978): "los conceptos siempre se están aprendiendo, modificando o haciendo más explícitos e inclusivos a medida que se van diferenciando progresivamente". Para evidenciar lo descrito anteriormente es necesario establecer comunicación con la estructura cognitiva del alumno manifestada en diversas 
formas tales como respuestas emitidas a diferentes interrogantes planteados con base en los criterios de puntuación establecidos en los trabajos de (Novak y Gowin, 1984).

\subsection{Dominio de conocimiento}

Para el estudio del Modelo del Átomo, el hipermedio se dividió en las siguientes unidades:

1. El origen del concepto en la Grecia Clásica.

2. El modelo Atómico de Thompson.

3 El modelo Atómico de Bohr.

4. El modelo Atómico de Rutherford.

5. El modelo Atómico de Sommerfeld.

6. El modelo de la Nube de Carga.

7. La teoría Mecanocuántica del modelo atómico.

\subsection{Aprendizaje colaborativo}

\subsection{Definición}

El aprendizaje colaborativo es un ambiente en el que los compañeros interactúan en un sistema educativo creando contextos sociales más agradables al tiempo que se aumenta la efectividad del sistema. Además, tal ambiente ayuda a sostener los intereses del estudiante y proporciona un hábitat de aprendizaje más natural que sirve para avivar y enriquecer el proceso de aprendizaje (Bouton and Garth, 1983; Alavi, 1994).

El aprendizaje colaborativo juega un papel importante en el desarrollo cognoscitivo constructivo, tal teoría es consistente con otras teorías de aprendizaje (Piaget, 1928, Piaget 1932, Vygotsky 1978, Fox \& Karen, Thomas \& Funaro 1990) en las que se enfatiza la importancia de la colaboración en el aprendizaje.

En el aprendizaje colaborativo se crea un ambiente en el que un estudiante actúa recíprocamente con los compañeros del grupo colaborando en la resolución de un problema dado. Se supervisan las interacciones entre los estudiantes y son controladas por el Sistema de aprendizaje colaborativo.

Un sistema de aprendizaje colaborativo se concentra en refinar e integrar el proceso de aprendizaje y el conocimiento de los estudiantes con la ayuda de los compañeros colaboradores. La promesa del aprendizaje colaborativo es permitir a los estudiantes aprender en contextos relativamente agradables y motivarlos cognoscitivamente y socialmente.

El estudiante puede discutir las estrategias para resolver un problema dado en un dominio de conocimiento con un grupo de estudiantes que aconsejan, motivan, critican, compiten, y dirigen hacia un mejor entendimiento de la materia.

\subsubsection{Las teorías del aprendizaje y la cognición en la colaboración}

Dillenbourg (Dillenbourg et al., 1994) identifica tres teorías diferentes de aprendizaje que podría emplearse en sistemas de aprendizaje colaborativo. Cada teoría relaciona el aprendizaje del estudiante con un ambiente típico de aprendizaje. Estas tres teorías se clasifican como desarrollos cognoscitivos que se enfocan en las interacciones entre los 
pares alrededor de las tareas apropiadas en un ambiente dado que incrementa el dominio de conceptos críticos.

Teoría socioconstructivista: Dice que los estudiantes dominan nuevos acercamientos de aprendizaje a través de la interacción recíproca con otros (Doise, 1990). Esta teoría es una extensión de la teoría de Piaget (Piaget, 1928; Piaget, 1932) que se enfoca en las razones del desarrollo cognoscitivo en los individuos. En la teoría del socioconstructivismo se da énfasis a las interacciones en lugar de las acciones. Un nivel determinado de desarrollo individual permite participación en ciertas interacciones sociales que producen nuevos estados individuales, que a su vez, hacen posibles interacciones sociales más sofisticadas (Dillenbourg et al., 1994). Los socioconstructivistas se acercan al enfoque del desarrollo del individuo con respecto a la interacción social, sin diferenciar o identificar realmente los factores subyacentes que refuerzan el aprendizaje colaborativo. Aquí la interacción social está supuesta como una caja negra que empuja al aprendizaje colaborativo.

La teoría sociocultural: Se enfoca en la relación causal entre la interacción social y el desarrollo cognoscitivo del individuo. Este acercamiento se deriva de la zona de desarrollo proximal de Vygotsky (Vygotsky, 1978). En este acercamiento cada cambio cognoscitivo interno es trazado por un efecto causado por una interacción social.

La teoría del conocimiento compartido: Difiere de las oras dos teorías en el sentido de que el ambiente en el que el aprendizaje toma lugar se da más por el enfoque que por los procesos cognoscitivos independientes del ambiente. El ambiente consiste en el contexto físico y el contexto social.

El conocimiento compartido apunta a que los pares aprendan conocimientos y habilidades en contextos donde ellos son aplicables (Brown, Collins, \& Duguid 1988, Lave \& Wenger, 1991). Algunas ventajas de ubicar el conocimiento son:

- Vinculando los contextos específicos y el conocimiento que va a ser aprendido, los pares aprenden bajo las condiciones en que el conocimiento debe aplicarse.

- Las situaciones desarrollan el pensamiento creativo. Los pares aprenden a menudo cómo puede aplicarse el conocimiento que ellos tienen en nuevas situaciones.

- El hecho de plantear situaciones a los pares conlleva a adquirir conocimiento de una forma más práctica y natural.

Según este acercamiento, la colaboración se ve como un proceso de construcción y mantener una concepción compartida de un problema, asegurando así un ambiente de aprendizaje natural.

\subsubsection{La enseñanza en el aprendizaje colaborativo}

Se identifican varias metodologías de enseñanza que son inherentes el apoyo del aprendizaje colaborativo

Práctica: Al par se le pide aplicar un objeto aprendido en un problema específico. 
Aprendizaje Socrático: El estudiante se inicia con una serie de preguntas sobre el dominio de conocimiento, a las que el estudiante reacciona con una hipótesis o con una pregunta que él posea.

Enseñanza-aprendizaje: Esta metodología apoya el aprendizaje donde el estudiante enseña al sistema, una variación en el uso del estudiante simulado. (Palthepu, Greer, \& McCalla, 1991) y (Nichols, 1994) han construido sistemas para apoyar la enseñanza en sistemas colaborativos.

Aprender de la situación: En esta metodología, el estudiante se convierte en un participante en una práctica sociocultural, donde las habilidades de aprendizaje y el proceso social van juntos (Wenger, 1988). El grado de la puesta en situación depende del ambiente que simula la colaboración.

Aprendizaje negociado: En esta metodología, el estudiante y el sistema negocian mutuamente las metas de aprendizaje aceptables (Moyse \& Elsom Cook, 1992, McCalla, 1990). El modelo del estudiante guarda huella de las metas de conocimiento mutuo.

\section{Aprendizaje por descubrimiento:}

El estudiante explora un ambiente especialmente creado para animarlo a aprender. Los pares podrían tomar papeles individuales en el ambiente de descubrimiento.

\section{METODOLOGÍA}

\subsection{Esquema del diseño experimental}

El desarrollo de la investigación estará compuesto por las siguientes fases:

FASE 1: Familiarización de los participantes en la investigación con el computador y la construcción de mapas conceptuales como un proceso de homogeneización de la muestra

FASE II: Estudio del hipertexto por parte de los estudiantes, Se escogen al azar para aplicar el diseño experimental que se describe en el numeral 6.2.

FASE III: Ejecución de una prueba para todos los grupos, consistente en un juego de escalera compuesto por un banco de 150 preguntas sobre los temas estudiados en el hipertexto "El Universo del Átomo". Las preguntas se presentan en tres formatos: respuesta única, respuesta múltiple y afirmación y razón, que se formularan al azar, si contestan afirmativamente les dará la posibilidad de hacer girar la ruleta para avanzar los pasos que en ésta logren". Con base en este juego se sacaran los puntajes que permitirán contrastar las hipótesis planteadas en el desarrollo de la investigación.

\subsection{Diseño experimental}

Se diseñaron dos versiones de software: Ambas cuentan con un núcleo básico compuesto por el hipertexto sobre el tema "Modelos del Átomo" una primera versión presenta además un módulo en el que los estudiantes deben construir los mapas conceptuales del tema que estudiaron de los siete que componen el hipertexto; la segunda, incluye un módulo simulador en el que se le presenta al estudiante el proceso de elaboración de un mapa que realiza un experto sobre uno de los siete temas que se 
plantean en el hipertexto; Estas dos versiones se combinan en dos ambientes sociales de aprendizaje por un lado el ambiente de aprendizaje colaborativo y del otro al ambiente de trabajo individual.

Las combinaciones de software y ambiente social de aprendizaje determinan cuatro condiciones experimentales para un diseño factorial dos por dos, como se representa en la siguiente tabla:

Tabla 1. Distribución de los grupos en el diseño experimental factorial 2 × 2 .

\begin{tabular}{|l|l|l|}
\hline \multirow{2}{*}{ MAPAS CONCEPTUALES } & \multicolumn{2}{|l|}{ AMBIENTE SOCIAL } \\
\cline { 2 - 3 } & COLABORATIVO & INDIVIDUAL \\
\hline CONSTRUCCIÓN & GRUPO A & GRUPO B \\
\hline OBSERVACIÓN & GRUPO C & GRUPO D \\
\hline
\end{tabular}

El diseño factorial dos por dos permitirá:

1. Al comparar los datos obtenidos de los grupos $A$ y $C$ contra los grupos $B$ y $D$ nos permitirá determinar si la segunda hipótesis se cumple, es decir si los grupos que trabajan en ambientes de aprendizaje colaborativo obtendrán mejores puntajes que los estudiantes que trabajan individualmente.

2. Al comparar los datos obtenidos por los grupos $A$ y $B$ contra los grupos $C$ y $D$ nos permitirá determinar la validez de la primera hipótesis: Obtendrán mejores resultados en una prueba final aquellos estudiantes que construyen mapas conceptuales frente a los estudiantes que observan la simulación de la construcción del mapa tradicional que realiza un experto en el estudio del concepto "Modelo del Átomo" en un ambiente hipermedial.

3. Al realizar el análisis cruzado de la matriz planteada en la tabla 1 se verificará cual de las condiciones combinadas ambiente social colaborativo y construcción de mapas es superior a la condición ambiente individual y observación de la simulación de la construcción de mapas conceptuales.

En el desarrollo de la condición experimental el componente social se trabajo de la siguiente forma: los sujetos de los grupos $B$ y $D$ trabajaron individualmente en una estación de trabajo. Los sujetos de los grupos $A$ y $C$ trabajaron en equipos de tres por estación de trabajo. La organización de tres obedece a los planteamientos de Blaye (Blaye et al. 1990) que establecen que la unidad mínima colaborativa debe ser tres ya que uno de los pares debe permitir el desequilibrio en la toma de decisiones.

\subsection{Descripción del software}

\subsubsection{El hipertexto}

El hipertexto esta compuesto de tres libros: el primero cuenta con 96 páginas (pantallas), las 40 primeras corresponden a la animación de la presentación de entrada del programa, las restantes corresponden a las siete unidades temáticas en las que se dividió el hipermedio para su estudio; las cuales son: El origen del concepto "Átomo", El 
modelo de Thompson, El modelo de Rutherford. El modelo de Bohr, El modelo de Sommerfeld, El modelo de la nube de carga, y El modelo Mecanocuántico.

El primer libro se llama "Atomo.exe". El segundo libro es el glosario del hipermedio y se encuentra en el archivo "orbital.exe", compuesto por 50 páginas; un tercer libro esta constituido por 18 páginas en las que se consigna la información del modelo de la Nube de Carga, el libro se denomina 'orb.exe".

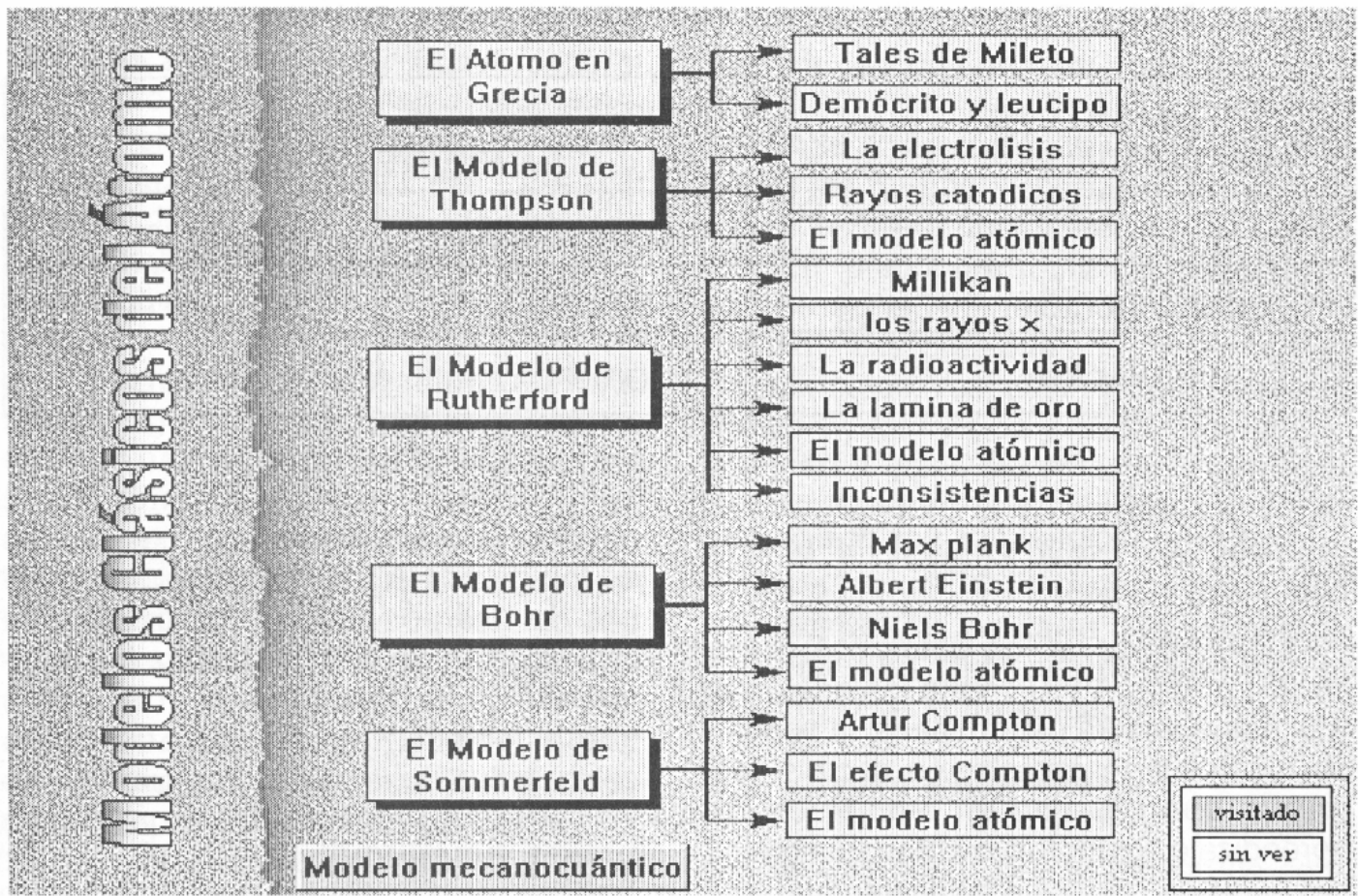

Gráfica 2. Mapa de navegación en el hipertexto correspondiente a los modelos clásicos del átomo.

\subsubsection{El módulo de construcción de los mapas conceptuales}

Este módulo cuenta con la paleta de herramientas, la barra de menú, el área de trabajo en la primera página. En la segunda página se va almacenando temporalmente la información de la construcción del mapa conceptual que esta elaborando el usuario. En la tercera página se encuentra la ayuda del programa.

\subsubsection{El módulo de simulación de los mapas conceptuales}

Cuenta además de los elementos ya planteados en el módulo de construcción de mapas conceptuales, con una paleta de control, compuesta por los botones de adelantar y retroceder en un cuadro la animación, el botón de play que permite observar todo el proceso de la simulación, el botón de velocidad que activa un visor en el que se muestra un dial con el que el usuario podrá controlar la velocidad con la que esta observando la simulación, en esta paleta también se encuentra un cuadro que muestra el número de eventos que tiene la simulación y al lado izquierdo la evolución de los eventos que se encuentran en el desarrollo de la simulación. 


\subsubsection{Las bases de datos}

El programa cuenta con dos bases de datos para almacenar la información, en ella se recogen los siguientes datos generales del usuario:

El código, la edad, el nombre, el sexo, el curso.

Para el interés de la investigación se recogen las páginas visitadas, los puntajes obtenidos en las seis evaluaciones. Los usuarios que deben elaborar mapas conceptuales cuentan con seis campos de registro para este fin. La segunda base corresponde al juego de a evaluación final y recoge el código de los usuarios, el pontaje obtenido y el tiempo utilizado.

\subsubsection{El juego final (la escalera)}

Este programa cuenta con una página principal en la que se encuentra el campo de juego constituido por cien cuadros enumerados con el número 1 hasta el 100 iniciando en el extremo inferior izquierdo y terminando en el extremo superior izquierdo. A lo largo del juego se encuentran obstáculos que hacen que el usuario descienda en casillas, los cuales se representan con los toboganes y los hoyos, o premios que le ayudan a avanzar y que se representan por las escaleras o por los helicópteros

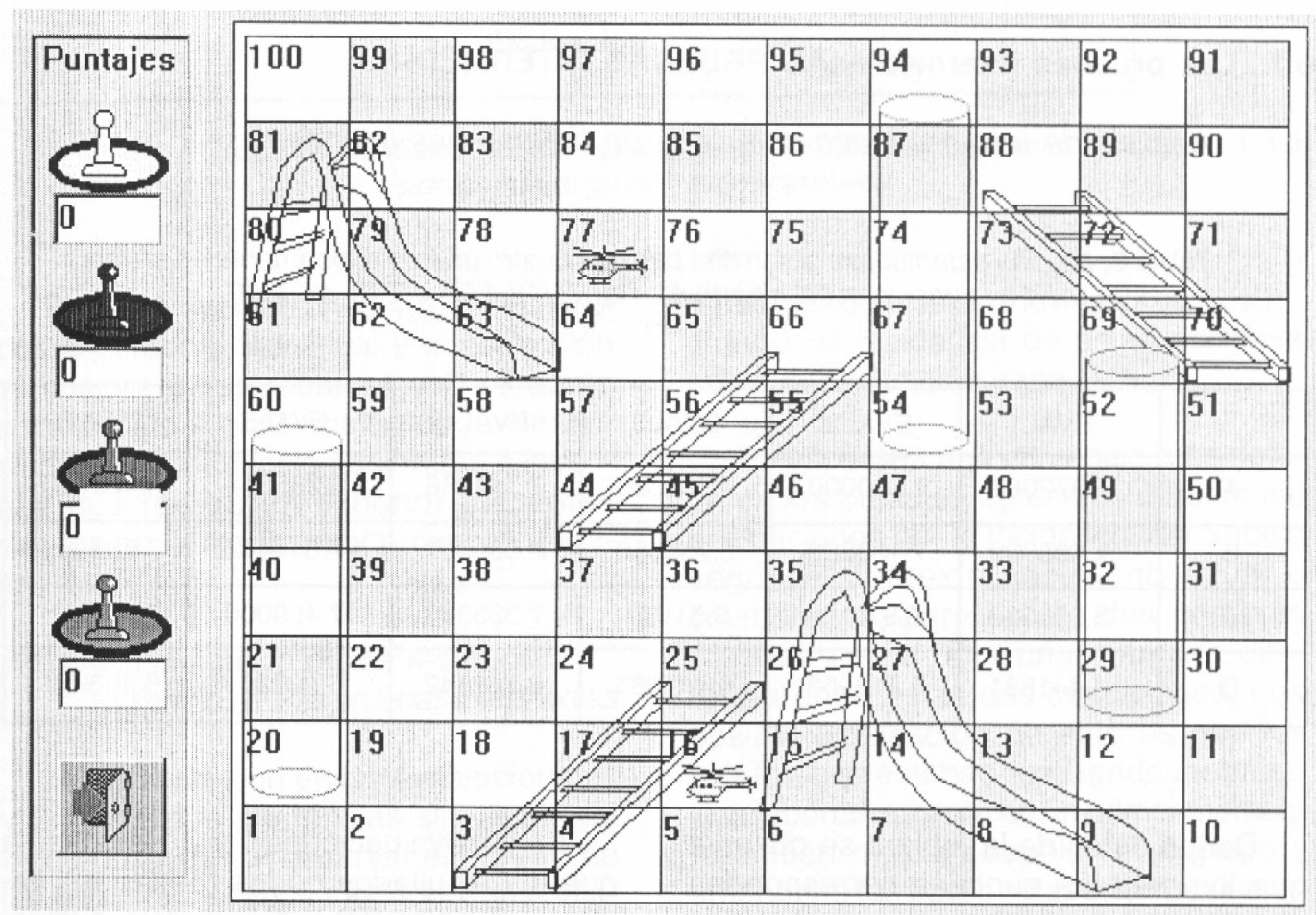

Gráfica 3. Pantalla del juego final.

\subsection{La población}

Se contó para el desarrollo del experimento con los alumnos del grado décimo del Colegio Distrital Bravo Páez, participaron 153 estudiantes, los cuales se distribuyeron aleatoriamente en las cuatro condiciones experimentales. La población está enmarcada en el rango de edades de 15 a 19 años. Es una muestra homogénea ya que todos 
contaban con la capacitación en el uso de los computadores, y además ya habían manejado mapas conceptuales en el desarrollo de las clases de Química, Español y sociales.

La distribución de estudiantes por grupo experimental fue la siguiente:

\begin{tabular}{|l|c|c|}
\hline \multirow{2}{*}{ MAPAS CONCEPTUALES } & \multicolumn{2}{|l|}{ AMBIENTE SOCIAL } \\
\cline { 2 - 3 } & COLABORATIVO & INDIVIDUAL \\
\hline CONSTRUCCIÓN & 37 & 40 \\
\hline OBSERVACIÓN & 39 & 37 \\
\hline
\end{tabular}

Tabla 2. Cantidad de estudiantes por grupo de diseño experimental

\section{ANÁLISIS E INTERPRETACIÓN DE RESULTADOS}

\subsection{Las pruebas intermedias PRUEBAS INTERMEDIAS}

6. 1.1 Análisis de los resultados obtenidos en las pruebas intermedias

Tabla 3. Medias estadísticas obtenidas para los cuatro grupos experimentales en las seis evaluaciones del hipertexto. Rao $R(18,472)=7.43, p<0000$.

\begin{tabular}{|l|l|l|l|l|l|l|}
\hline & EVAL 1 & EVAL 2 & EVAL 3 & EVAL 4 & EVAL 5 & EVAL 6 \\
\hline A & 4.372093 & 7.000000 & 7.302326 & 7.744186 & 8.255814 & 8.162790 \\
\hline B & 4.48889 & 7.133333 & 6.755556 & 7.066667 & 7.644444 & 7.688889 \\
\hline C & 3.933333 & 6.911111 & 6.511111 & 7.333333 & 7.400000 & 7.777778 \\
\hline D & 4.441861 & 6.813953 & 6.441861 & 6.767442 & 6.837209 & 6.906977 \\
\hline
\end{tabular}

De los datos de la tabla 3 se observa que los mejores puntajes corresponden al grupo A que tiene como condiciones experimentales la Construcción de los mapas conceptuales y del trabajo colaborativo, en tanto que los pontajes más bajos corresponden al grupo $D$ que tiene como condiciones experimentales la observación de la simulación de la construcción del mapa conceptual que trabaja un experto y la condición de ambiente de trabajo individual. Igualmente es de notar que los grupos B y $\mathrm{C}$ presentan pontajes en la media muy cercanos.

En las evaluaciones 1 y 2 se observa que los resultados de las medias se encuentran entre 3,9 y 4,5 para la evaluación 1; siendo los pontajes del grupo C los más bajos, y en la evaluación 2 entre 3,0 y 3,1; siendo casi equivalentes para los cuatro grupos.

Desde la evaluación 3 hasta la evaluación 6 se nota un cambio en la curva de medias siendo mayor la del grupo A en tanto que la menor corresponde al grupo D. 


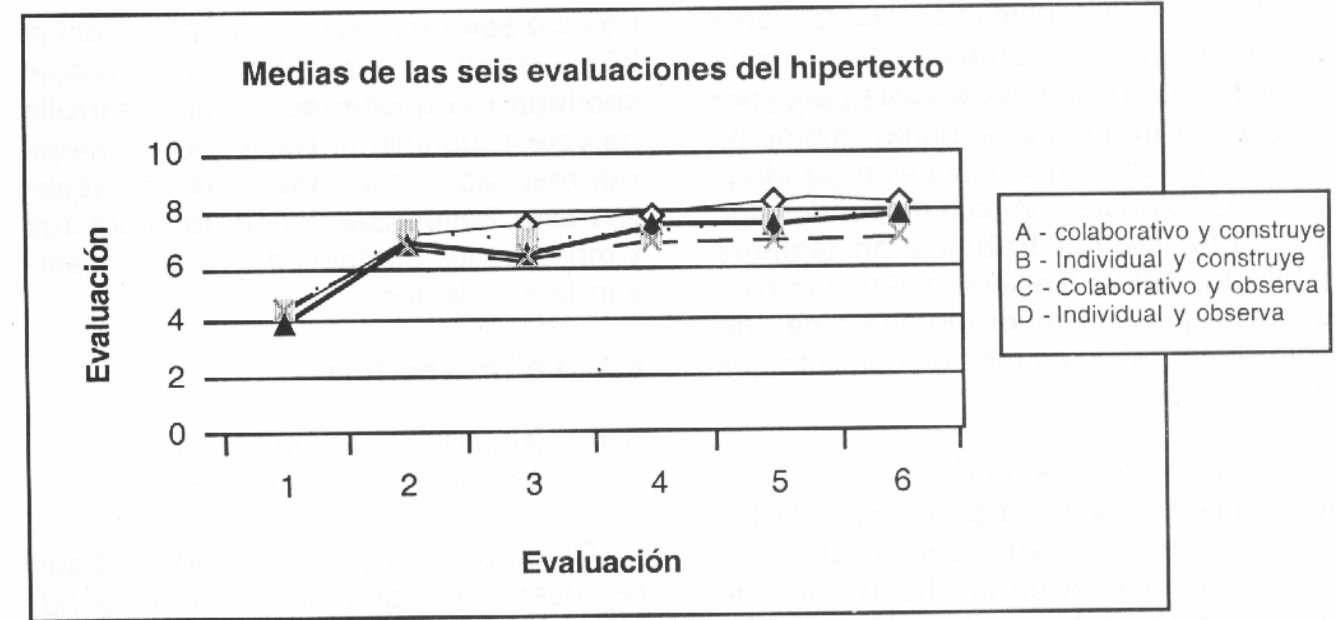

Gráfica 4. Medias de las seis evaluaciones del hipertexto obtenidas por los cuatro grupos experimentales.

Para las evaluaciones 4,5 y 6 los grupos B (Trabajo individual y construcción de mapas conceptuales) y $\mathrm{C}$ (Trabajo colaborativo y observación de la simulación de la construcción del mapa conceptual que realiza un experto) presentan valores en la media muy próximos. (Gráfica 4).

\subsubsection{Interpretación de los resultados obtenidos en las pruebas intermedias}

Se observa que en las evaluaciones 1 y 2 no existen diferencias significativas, es de notar que al observar el registro de las páginas visitadas casi todos los sujetos inician una exploración lineal del hipertexto, iniciando la observación con las unidades 1 y 2 del hipermedio. En esta etapa la influencia de la construcción de mapas conceptuales no es evidente porque la tarea que realizan los usuarios es más de exploración que de un trabajo concienzudo de por sí. La construcción u observación de la simulación de la construcción de los mapas conceptuales es una tarea que se realizaba después de haber contestado la evaluación, por lo que no se puede explicar los valores obtenidos por la incidencia de estas acciones sobre la evaluación inmediata.

Se esperaba una influencia más notoria en los puntajes obtenidos durante las evaluaciones perla incidencia del trabajo según el contexto social Individual o colaborativo, pero en las dos primeras pruebas no se nota una marcada diferencia entre estas dos condiciones, una posible explicación de esto es que los sujetos que estaban realizando su trabajo colaborativamente en un primer momento actúan más en acoplarse al grupo y entender a los pares colaborativos que en la realización efectiva de tareas. Es de anotar que la organización de los grupos colaborativos se realizó aleatoriamente y a pesar de que eran sujetos pertenecientes a un mismo curso de estudio solamente están acostumbrados a trabajar con un determinado grupo y ante la situación de conformar un nuevo grupo de trabajo su primera tarea es de acople en el grupo, Esto es consistente con los planteamientos de Blaye y otros (Blaye et al., 1991) quienes consideran como tarea crucial la constitución de los pares colaborativos y que en esta investigación no se pudieron establecer estos pares por conveniencia, siendo el primer momento de la evaluación una discusión de ideas y no una colaboración para responder algo acertado. De los trabajos de Galliers (Galliers, 1988; Galliers, 1989) también destaca que un primer momento del trabajo colaborativo es más de conflicto que de acuerdos. 
Cuando se ha superado la etapa de los conflictos se llega a la etapa de los acuerdos en los pares colaborativos, y esto se evidencia en la observación de los datos obtenidos en la gráfica 4 y ja tabla 3 donde se observa que los mejores pontajes en las evaluaciones 4,5 , y 6 corresponden a los grupos que trabajan colaborativamente

La igualdad que se puede observar en las 3 evaluaciones últimas del hipertexto entre los grupos B y C muestra que es equivalente en el desarrollo de este trabajo el aprendizaje colaborativo y la construcción de mapas conceptuales.

Luego de haber observado que la construcción del mapa conceptual demanda un cuidado superior en el estudio del hipertexto los sujetos se dedican a estudiar el material con mayor detenimiento en tanto que los sujetos que tienen la observación de la construcción del mapa conceptual realizado por un experto no presentan el mismo interés, ya que su trabajo requiere menos trabajo que el que requiere el que esta construyendo el mapa conceptual. Estas observaciones son acordes con los trabajos realizados por Menn (Menn 1993) quien evaluó el impacto de medios de comunicación instruccionales diferentes en la retención del material de estudio y encontró que: los estudiantes recuerdan solamente el $10 \%$ de lo que ven, $50 \%$ si observan a alguien que este realizando un trabajo aún cuando esto sea una simulación, pero casi el 900 o si ellos hacen el trabajo, Lo cual confirma lo encontrado en el desarrollo de este experimento en el que los mejores resultados son obtenidos por aquellos estudiantes que tienen que construir y menores los pontajes de los que observan la simulación.

\subsection{La prueba final}

\section{1. 1 Análisis de los resultados obtenidos en la prueba final}

Cuando los sujetos habían acabado de observar el hipertexto y una vez se hubieran contestado las seis evaluaciones se enfrentaban a un juego de escalera, en éste la condición para poder avanzar era el contestar correctamente la pregunta que el sistema le seleccionaba al azar en un banco de 150 preguntas estilo ICFES de respuesta única, afirmación y razón y selección múltiple.

\begin{tabular}{|l|c|}
\hline GRUPOS & PROMEDIO \\
\hline A & .685860 \\
B & .528311 \\
C & .635289 \\
D & .447581 \\
All Grps & .574432 \\
\hline
\end{tabular}

Tabla 5. Tabla de medias para la prueba final.

De la gráfica 5 y la tabla 5 se observa que: Los mejores pontajes corresponden a los grupos $A$ y $C$ y los más bajos son los de los grupos $B$ y $D$, los primeros son los que trabajaron en ambientes colaborativos, en tanto que los mas bajos son los de los sujetos que trabajan en ambiente individual.

Es de notar que el grupo B es superior al grupo D, ambos en ambiente de aprendizaje individual, pero, los sujetos del grupo B construían los mapas conceptuales, en tanto que los del grupo D observaban la construcción del mapa conceptual, igual relación se nota entre las medias de los grupos $\mathrm{A}$ y $\mathrm{C}$ que trabajaron en ambiente colaborativo, pero el mayor pontaje corresponde al grupo A que es el que construía el mapa en tanto que los 
sujetos del grupo O observaban la simulación de la construcción del mapa conceptual realizado por un experto.

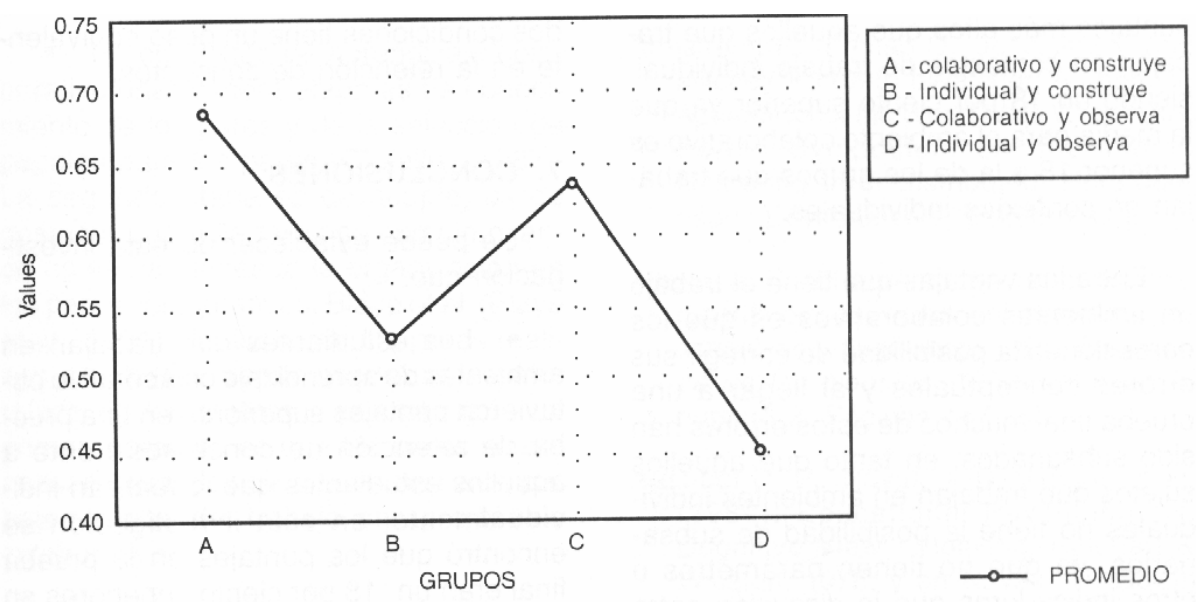

Gráfica 5. Medias de los cuatro grupos experimentales en la prueba final

Tabla 6. Análisis de Varianza para la prueba final.

Marked effects are significant at $p<.05000$

\begin{tabular}{|c|c|c|c|c|c|c|c|c|}
\hline & SS Effect & $d f$ Effec & MS Effect & SS Error & df Error & MS Error & F & $p$ \\
\hline PROM. & 1.4881 & 3 & 49606 & 11.390 & 172 & .06622 & 7.4906 & .00009 \\
& 99 & & 6 & 59 & & 4 & 91 & 7 \\
\hline
\end{tabular}

El valor de $p=0.000097$ en la tabla 6 muestra que los datos obtenidos son significativos, lo que indica una marcada diferencia entre los cuatro grupos experimentales debida a la condición experimental.

\subsubsection{Interpretación de los resultados obtenidos en la prueba final}

De la gráfica 5 y la tabla 5 se observa que la influencia experimental con la cual se obtuvieron los mayores pontajes en la prueba final de retención de conceptos corresponde a los grupos $A$ y $D$, lo cual muestra que en las dos condiciones experimentales de base mapas conceptuales y ambiente social de trabajo es más fuerte la segunda.

De la tabla 5 se observa que después de pasar la etapa de acople del grupo los que trabajan colaborativamente obtienen pontajes más altos que aquellos que trabajan en contextos de trabajo individual, siendo un 18 por ciento superior ya que la media para el ambiente colaborativo es superior 18 a la de los grupos que trabajan en contextos individuales.

Entre las ventajas que tiene el trabajo en ambientes colaborativos es que los pares tienen la posibilidad de corregir sus errores conceptuales y al llegar a una prueba final muchos de estos errores han sido subsanados, en tanto que aquellos sujetos que trabajan en ambientes individuales no tiene la posibilidad de subsanarlos, ya que no tienen parámetros u otros indicadores que la discusión entre los pares colaborando si brindan. 
Un sistema de aprendizaje colaborativo se concentra en refinar e integrar el proceso de aprendizaje y el conocimiento de los estudiantes con la ayuda de los compañeros colaboradores (Smith 1994).

En la condición experimental de los mapas conceptuales la retención de conceptos es superior para los sujetos que construyen los mapas conceptuales frente a los que observan la construcción del mapa conceptual en una simulación. En los pontajes obtenidos en la prueba final se encuentra que los que construyeron los mapas conceptuales están un 6 por ciento por encima de aquellos que solamente observan la simulación de la construcción del mapa conceptual. Esto es acorde con los planteamientos de Menn (Menn 1993) quien afirma que aquel que construye obtiene un porcentaje mayor de retención de un material de estudio que aquellos que observan una simulación.

Es de notar que combinando las dos condiciones al realizar el estudio diagonal de las medias no se encuentra una diferencia significativa entre las medias, lo cual indica que la combinación de las dos condiciones tiene un peso equivalente en la retención de conceptos.

\section{CONCLUSIONES}

Se puede establecer de esta investigación que:

Los estudiantes que trabajan en ambientes de aprendizaje colaborativo obtuvieron pontajes superiores en una prueba de retención de conceptos frente a aquellos estudiantes que lo realizan individualmente, en esta investigación se encontró que los puntajes en la prueba final eran un 18 por ciento superiores en la media.

En la condición experimental de la construcción frente a la observación de la simulación se encuentra que los sujetos que realizaban la construcción de mapas conceptuales tenían en la media un 6 por ciento de pontajes superiores a los que solamente observaban la simulación de la construcción de los mapas conceptuales que había realizado un experto.

Con los resultados observados se determiné que la primera hipótesis (hipótesis nula) no cumplía su predicción en tanto que la hipótesis 2 si se afirmaba con los datos obtenidos, ya que se confirmé que los resultados obtenidos por los estudiantes que trabajaban colaborativamente eran superiores a los puntajes obtenidos por los sujetos que estudiaron el hipertexto individualmente.

- Con los resultados obtenidos se confirmé ja hipótesis 4 y se descarté la hipótesis 3 , ya que se determiné de los resultados obtenidos en esta investigación que los estudiantes que construyen mapas conceptuales obtienen pontajes superiores a los sujetos que observan la simulación de la construcción de los mapas conceptuales que había realizado un experto.

- En contextos de aprendizaje colaborativo una primera etapa es de conocimiento de los pares y de la selección de papeles para los pares (O’Malley, 1987). La segunda etapa es de acople, es de destacar que esta tarea se cumple cuando no se ha planeado la organización de los pares colaborativos. Blaye et al (Blaye et al., 1991) plantean que los pares realizan sus tareas dependiendo de la naturaleza de los sujetos y por tal razón un 
primer momento es que los pares se conozcan para decidir cuales son sus papeles dentro del trabajo en aprendizaje colaborativo.

- Después de realizar las etapas de reconocimiento de papeles y acoplamiento el trabajo colaborativo es más efectivo porque los estudiantes pasan de la fase de conocimiento del par y conflicto (Galliers, 1989) y se pasa a la etapa de integración del aprendizaje (Smith, 1994) y su influencia en la retención de conceptos es más llamativa para los pares que trabajan colaborativamente frente a los sujetos que estudian el hipermedio en contextos de trabajo individual.

- La combinación de construcción de mapas conceptuales en ambientes colaborativos es superior en un 22 por ciento contra la observación de la simulación de la construcción de los mapas conceptuales en ambientes de trabajo individual, las dos variantes contexto colaborativo y construcción de mapas demuestra su eficacia, ya que el que trabaja esta haciendo efectivo el aprendizaje constructivo en el que se demuestra que la retención conceptual es mayor cuando se realiza la tarea que cuando se observa la construcción de tal tarea esto es acorde con los estudios de Menn (Menn 1993)

\section{REFERENCIAS}

Armstrong R., Freitag D., Joachims T. And Mitchell T. (1995) bWatcher: A Learning Apprentice for the World Wide Web'. AAAl Spring Symposium on Information Gathering from Distributed, Heterogeneous Environments, Stanford, CA.

Astolfi, J. P. "El aprendizaje de conceptos científicos: aspectos epistemológicos, cognitivos y lingüísticos". En: Enseñanza de las ciencias, 1988, volumen 6, número 2. Páginas 147-155.

Ausubel D. P., Novak J. D., Hanesian H. (1984). Educational Psychology: A Cognitive View - HoltHolt, Rinehart \&Winston, 1978; y en Novak JD, Gowin DB - Learning how to Learn, Cambridge U. Press.

Ausubel, David. (1978). Psicología educativa, un punto de vista cognitivo. México: ed. trillas.

Baecker R.M., Grudin J., Buxton W.A.S., Greenberg S. (1995). Hypertext and multimedia. Human-Computer Interaction: Toward the Year 2000, Chapter 13, pp. 833-842.

Bautista, O. y Guerrero, G. La enseñanza de las ciencias: difusión de valores científicos. Rey. Física y cultura. UPN. Bogotá; 1(2):15-20. 1990.

Blaye, A. (1989). Confrontation sociocognitive et resolution de problemes. Doctoral dissertation, Centre de Recherche en Psychologie Cognitive, Université de Provence, 13261 Aix-en-Provence, France.

Blaye, A., Light, P., Joiner, R., \& Sheldon, S. (1990). Collaboration as a facilitator of planning and problem solving on a computer based task. British Journal of Psychology, 9, 471-483.

Briggs, L. J. (1968). Sequencing of Instruction in Relation te Hierarchies of Competence. American Institutes ter Research, Pittsburgh. PA. 
Brown Kappe, Collins Andrews and Dugui Schipflinger (1988). A new tool for distributed hypermedia. In International Conference en Distributed Multimedia Systems and Applications, Honolulu, Hawaii.

Brown A.L. \& Collins (1988). Reciprocal Teaching of Comprehension-Fostering and Comprehension-Monitoring Activities. Cognition and Instruction, vol.1, nxbc 2, pp. 117175.

Brown J. S and Burton R. R. (1978). Diagnostic models for procedural bugs in basic mathematical skills. Cognitive Science, vol. 2, pp. 155-92.

Chan, G. P. \& Baskin, E. M. (1988). "Cooperative learning and achievement: Methods for assessing causal mechanism." Ln S. Sharan (Ed.), Cooperative learning, theory and research (pp. 1-22). New York: Prager.

Chan, O. P. (1991). Cooperative learning in the classroom: Research in esegregated schools. Hillsdale, NJ: Erlbaum.

Corredor, Martha. "Autotext: un sistema Hipertexto para autoría de material educativo computarizado". En: Informática Educativa, Vol. 4, núm. 3,199], páginas 247-253.

Dansereau, D. F. (1988). Learning from lectores: Effects of cooperative review. Journal of Experimental Education, 61(2), 116-125.

Dillenbourg, P., Baker, M., Blaye, A. \& O'Malley, C. (1994). The evolution of research en collaborative learning. In E.Spada \& P. Reiman (Eds) Learning in Humans and Machines

Doise, W. (1990). The social development of the intellect. Oxford: Pergamon Press.

Fox, B. \& Karen, H. (1990). Interactional reconstruction in real-time language processing. Cognitive Science, 11 (3), 365-387.

Gil, Daniel y Paya, José. Los trabajos prácticos en la enseñanza de la física y la química. España. 1990.

Gil, Daniel. "La metodología científica y la enseñanza de las ciencias. Unas relaciones controvertidas". Rey. Enseñanza de las ciencias. Madrid. 4(2). Páginas 111-121. 1986.

Goodlad, John. (1996). Un nuevo concepto de programa escolar. Madrid: Editorial magisterio Español.

Kuhn, C. G. (1972). Archetypes of the collective. In The Basic writings of CG. Jung. ed: V. S. De Laszlo. New York: The Modern Library.

Maldonado, L. F.; Fonseca, O.; Ibáñez, J.; Macías, D.; Ortega, N.; Rubio, MR.; y Sanabria, L. B. (1999). Metacognición y razonamiento espacial en juegos de computador. Bogotá: Universidad Pedagógica Nacional - Instituto para la Investigación Educativa y el Desarrollo Pedagógico, U.P.N. - DEP.

Miyake, N. (1986). Constructive Interaction and the Iterative Process of Understanding. Cognitive Science, 10, 151-177. 
Monroy Henao, Betty. Procesos cognitivos, CIDUP. Bogotá, 1994. Páginas 57-63,

Novack, J. y Gowin D. (1984). Aprendiendo a aprender. Barcelona: Ediciones Martínez Roca.

Novack, J. D (1988). Constructivismo humano: un consenso emergente. Revista de enseñanza de las ciencias, Vol 6, No 3, 1988.

Rogoff, B. (1991). Social interaction as apprentice ship in thinking: guided participation in spatial planning. In L.Resnick, J. Levine \& S. Teasley (Eds). Perspectives en Socially Shared Cognition (pp. 349-364). Hyattsville, MD: American Psychological Association.

Rolston, David. (1995). Principios de inteligencia artificial y sistemas expertos. México: Editorial McGraw Hill.

Rossi, Gustavo Héctor. "Sistemas de Hipermedia: una nueva filosofía para crear y tener acceso a bases de información". En: Revista Informática Educativa, Vol. 4 . No 3. Bogotá, 199]. Páginas 207-218.

Slavin, R. E. (1985). "Team-assisted individualization: Combining cooerative learning and individualized instruction in mathematics." In RE. Slavin, S. Sharan, S. Kagan, R. HertzLazarowitz, C.Webb \& R. Schmuck (Eds.), Learning to cooperate, cooperating to learn, (pp. 177-209). New York: Plenum.

Slavin, R. E. (1990). Using student team learning. Baltimore, MD: Center for Social Organizations of Schools, Johns Hopkins University.

Smith, J. J. (1994, August). The SPICE project: Comparing passive to interactive approaches in a videodisc-based course. Technologicai Horizons in Education Journal, 21(1), 62-66.

Thomas L. \& Funaro, S. (1991) Learning Oonversations. New York: Reuteledge. New York: Pergamon Press.

Van Lehn, W. \& Ohlson, B. S. (1994). Collaborative learning and social organization among students. Teaching Sociology, 18.

Vygotsky, L. S. (1962). Thought and Language. Cambridge, Massachusetts: MIT Press.

Vygotsky, L. S. (1978), Mmd in Society. The Development of Higher Psychological Processes. Edited by M. Cole, V. JohnSteiner, S. Scribner \& E. Seuberman Harvard University Press. Cambridge, Press. 